\title{
Solving the ECGI Problem with Known Locations of Scar Regions
}

\author{
M Malal Diallo ${ }^{1,2,3}$ Mark Potse $^{1,2,3}$ Rémi Dubois $^{1,4,5}$ Yves Coudière $^{1,2,3}$ \\ ${ }^{1}$ IHU-Liryc, Fondation Bordeaux Université, Pessac, France \\ ${ }^{2}$ CARMEN Research Team, Inria Bordeaux - Sud-Ouest, Talence, France \\ ${ }^{3}$ Univ. Bordeaux, IMB, UMR 5251, CNRS, Talence, France \\ ${ }^{4}$ Univ. Bordeaux, CRCTB de Bordeaux, U1045, Bordeaux, France \\ ${ }^{5}$ INSERM, CRCTB, U1045, Bordeaux, France
}

\begin{abstract}
We propose a methodology to take into account the location of scars in ECGI problem. The method is to consider the whole body, including blood, heart and remaining volume as a conductor with an electric current source field localized in the heart. We identify the source best matching a given body surface potential map, by solving the classical quadratic optimization problem with a Tikhonov regularization term. The method behaves better than the MFS method in presence of a scar. The correlation coefficients of the activation times around the scar are improved up to $10 \%$ on the epicardium, and $7 \%$ on the endocardium, by adapting the Tikhonov regularization parameter and conductivity coefficient in the scar.
\end{abstract}

\section{Introduction}

Electrocardiographic imaging (ECGI) is a non-invasive technique that is used to reconstruct the electrical activity of the heart from body surface electrical potential maps (BSPM), and the geometry of the heart and torso. The most common approach to compute this reconstruction is based on the model of the torso as a passive volume conductor, outside the heart. Hence the Laplace equation is set on the volume between the epicardium and the body surface. The method of fundamental solutions (MFS) with Tikhonov regularization is commonly used to solve the corresponding ECGI problem [1]. In clinical care, structural images of the patient are often available. A major question is therefore how to integrate this information in order to drive the inverse problem. It is limited because it cannot easily take into account scars inside the heart volume, and also because it assumes that cardiac sources are only distributed on the epicardium.

In this paper, we consider the torso as a volume conductor including the intracavitary blood, the heart and the remaining torso volume, in which only the heart volume behaves as an electric current source field. Hence we try to reconstruct the cardiac electrical volume source, and we can take into account a different electrical conductivity in each of the regions, and in particular in the scar. We discretize the equation with a standard finite element method, and apply the same Tikhonov regularization technique as with the MFS. We will refer to this method as the volume method (VM). In order to account for scars, we propose to increase the regularization parameter in the scar, which is possible with both the usual MFS and the proposed VM, but also to decrease the conductivity in the scar, which is possible only with the VM method.

Datasets computed on a realistic human-based anatomical model [2] were used to evaluate the method. The activation times (ATs) recovered by the standard MFS and VM method were compared to the reference ATs obtained from the model. We found that weighting the regularization parameter and decreasing the electrical conductivity by a factor 10 in the scar improved the correlation between the estimated and the true ATs, especially near the scar.

\section{Methods}

\subsection{Mathematical model}

The mathematical model consists of the static Laplace equation for the potential field $u(t, x)$ in the blood, heart, and torso domains, respectively denoted by $\Omega_{B}, \Omega_{H}$, and $\Omega_{T}$ (figure 1). It reads

$$
-\nabla \cdot(\sigma(x) \nabla u(t, x))=F(t, x) \quad \text { in } \Omega,
$$

(where $\Omega=\Omega_{B} \cup \Omega_{H} \cup \Omega_{T}$ ) with the no flux boundary condition $-\sigma \nabla u \cdot n=0$ on the torso surface $\partial \Omega$. In this model, the electrical conductivity is a function $\sigma(x)$, piecewise constant given below, and the source term $F(t, x)$ accounts for the total ionic current. The conductivity function is 
given by

$$
\sigma(x)= \begin{cases}\sigma_{H} & \text { if } x \in \Omega_{H} \backslash \Omega_{\mathrm{scar}}, \\ \rho \sigma_{H} & \text { if } x \in \Omega_{\mathrm{scar}}, \\ \sigma_{B} & \text { if } x \in \Omega_{B} \\ \sigma_{T} & \text { if } x \in \Omega_{T}\end{cases}
$$

where the factor $\rho \leq 1$ may be used to decrease the conductivity in the scar areas; and the source term is

$$
F(t, x)= \begin{cases}f(t, x) & \text { if } x \in \Omega_{H}, \\ 0 & \text { if } x \in \Omega_{B} \cup \Omega_{T} .\end{cases}
$$

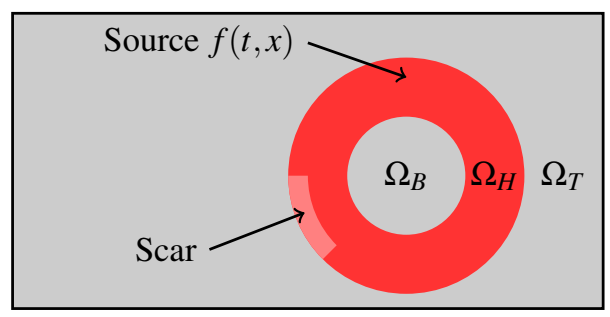

Figure 1. Sketch of the model with a scar.

Thus, the geometry and the conductivity coefficients are parameters of our model. The body surface potential map is the trace of $u(t, x)$ on the boundary of $\Omega$, denoted by $u_{T}:=u_{\mid \partial \Omega}$. The extracellular potential is the restriction of the potential $u$ to the cardiac domain, $u_{\Omega_{H}}$. At each time instant, the forward problem maps the source $f(t, x)$ to the BSPM $u_{T}(t, x)$, while the inverse problem consists in finding an inverse mapping from BSPM data $d$ to a source term $f$ in the heart, which direct image best matches $d$.

\subsection{Numerical resolution}

Equation (1) was discretized by the P1 Lagrange finite element method using the FreeFem++ software [3]. The matrix of the direct problem, called the transfer matrix, is an $N_{T} \times N_{H}$ matrix denoted by $M$, such that $u_{T}=M f$ where $N_{T}$ is the numbers of mesh nodes on the body surface $\partial \Omega$, and $N_{H}$ the number of mesh nodes in the heart domain $\Omega_{H}$.

We used the Tikhonov regularization method, because the inverse problem is ill-posed and underdetermined. In order to account for the scar, we

- increased the regularization weight in the scar areas, which amounts to forcing the source $f$ towards 0 in these areas; and

- decreased the electrical conductivity in the scar by the factor $\rho$ (equation (2)).

Given data $d \in \mathbb{R}^{N_{T}}$ on all torso nodes, we reconstructed the source term $f \in \mathbb{R}^{N_{H}}$ as the optimum

$$
f=\operatorname{argmin}_{g \in \mathbb{R}^{N_{H}}}\left\{\|M g-d\|^{2}+\lambda^{2}\|W g\|^{2}\right\},
$$

where $W$ is a diagonal weight matrix defined by $W_{i i}=w \geq$ 1 if the degree of freedom number $i$ is in the scar, and $W_{i i}=$ 1 otherwise. $w$ is called the regularization weight. The solution of problem (4) is finally computed by solving the normal equation

$$
\left(M^{T} M+\lambda^{2} W^{T} W\right) f=M^{T} d .
$$

The regularization parameter $\lambda$, which stabilizes the solution $f$, was chosen using the L-curve method.

\subsection{Simulated data and evaluation}

Validation data were simulated with a monodomain reaction-diffusion model of the heart with a finite difference method on a mesh of $0.2 \mathrm{~mm}$ resolution. Computed transmembrane currents were transferred to a torso model with $1 \mathrm{~mm}$ resolution to compute torso potentials [2]. Scar zones were simulated by absence of transmembrane current, zero conductivity in the intracellular domain, and isotropic conductivity in the extracellular domain. The datasets used here correspond to two scenarios: i) Four pacing sites with a single scar area; and ii) a single pacing site without scar.

Reference activation times (ATs) of the simulated data were computed directly from the values of the transmembrane voltage. Using the simulated BSPM, extracellular cardiac potentials were computed with the MFS and an increased weight in the scar. Using the same data, source terms were computed with the VM, an increased weight, and decreased conductivity in the scar. Afterwards, ATs were calculated using the method proposed by Duchateau et al. [4], directly for the MFS, and after solving equation (1) to recover the extracellular potential $u_{\mid \Omega_{H}}$ for the VM. Correlation coefficients (CC), and relative errors (RE) between the reconstructed ATs and the reference ones were computed.

For computations, the weights ranged from 1 to 10 , and for the VM the conductivity coefficient was, in addition, multiplied by the fixed factor $\rho=0.1$.

Both MFS and VM ATs were compared to the reference ATs on the epicardium. The VM ATs were also compared to the reference ATs on the endocardium. In presence of a scar, the ATs are not computed in the scar zone.

\section{Results}

\subsection{Single pacing site without scar}

At first, we used data computed in absence of scar, and with a single pacing site in the heart, located near the apex. In this case, the weight $W$ is the identity matrix, and the conductivity coefficient $\sigma$ is constant in the whole heart. The results are presented in figures 2 and 3 , and in table 1 . 


\begin{tabular}{lllll}
\hline & \multicolumn{2}{c}{ Epi } & \multicolumn{2}{c}{ Endo } \\
& CC & RE & CC & RE \\
\hline MFS & 0.90 & 0.16 & - & - \\
VM & 0.89 & 0.17 & 0.67 & 0.29 \\
\hline
\end{tabular}

Table 1. CC and RE for the MFS and VM, single pacing site, no scar.

A
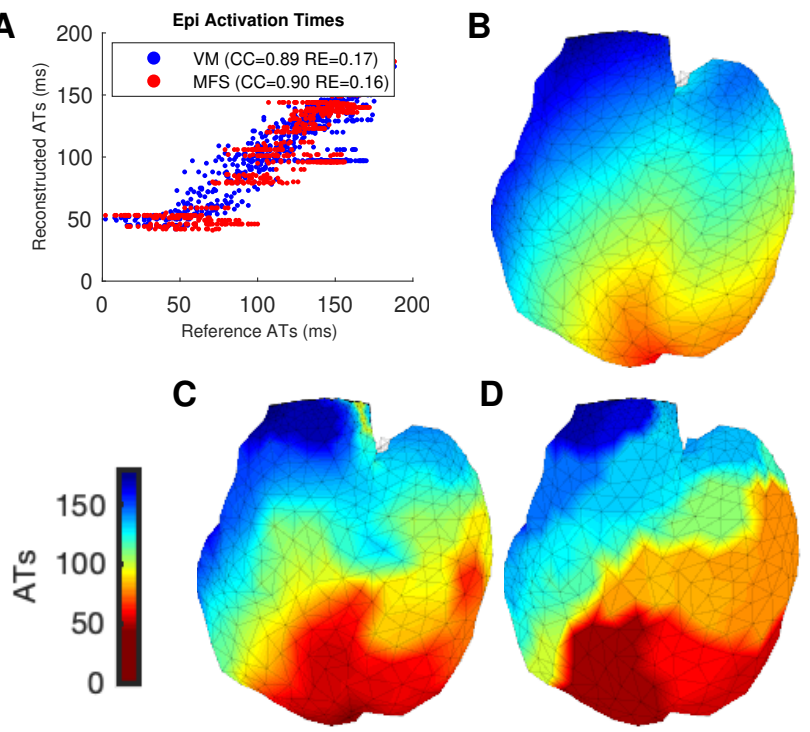

Figure 2. Epicardium, single pacing site, no scar. A: reconstructed ATs versus reference ATs, MFS in red and VM in blue. B: simulated ATs. C: ATs reconstructed by VM. D: ATs reconstructed by MFS.

The CC and RE of the VM method remained close to those of MFS (table 1), and its ATs map was smoother than the MFS map (figure 2). Furthermore, the VM method could reconstruct the endocardial map (figure 3 ).

\subsection{Four pacing sites with scar}

Next we used data computed in presence of a left ventricular scar, and four pacing sites in the heart. In this case, we varied the weight $w$ between 1 and 10, and decreased the conductivity by a fixed factor 10 in the scar for the VM. The results are presented in figures 4 and 5 , and table 2 .

In Figure 6, we show how the CC related to the weight $w$, all computed only in the neighbourhood (two layers of triangles) of the scar.

The CC and RE are degraded similarly for both methods (with respect to the absence of a scar). The VM resulted in smoother ATs than the MFS (figure 4). The CC computed only in the neighborhood (two layers of triangles) of the scar varied slightly with respect to the weight for the MFS, while it clearly improved for the VM, and improved even more when the conductivity was decreased by a factor
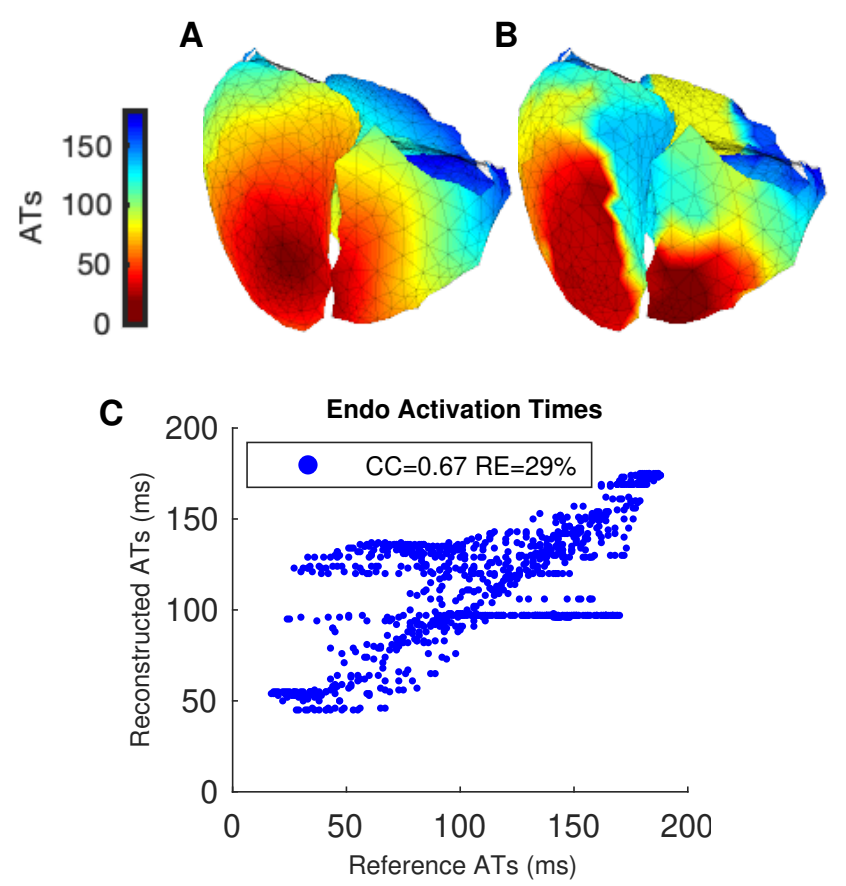

Figure 3. Endocardium, single pacing site, no scar. A: simulated ATs. B: ATs reconstructed by VM. C: reconstructed ATs versus reference ATs.

10 , up to $10 \%$ on the epicardium, and $7 \%$ on the endocardium (figure 6). The weight that maximized the $\mathrm{CC}$ was near 5 on the epicardium, and 2 on the endocardium. This suggests that it might be interesting to decrease the weight from the epicardium to the endocardium (in the scar).

\section{Discussion and conclusions}

Our objective was to use prior information on scar location in solving the ECGI problem, so as to reconstruct ATs from BSPM. To this aim, we proposed a volume method, which reconstructs a volumetric whole-heart source, instead of the epicardial potential field used in the MFS. The VM is quite different from the MFS since it directly looks for an current source field instead of a potential, and also because it accounts for the electrical conductivity in the heart, and its variation in a scar, but also in the intracavitary blood, while the MFS only accounts for the torso conductivity.

For the two examples presented in section 3, the VM performed as well as the MFS in the absence of scar, and better than the MFS in the presence of a left ventricular scar. In addition, the VM could reconstruct the endocardial activation (figure 3 and figure 5).

The activation in the presence of a scar is usually hard to reconstruct with classical ECGI methods. Our results may help to overcome this difficulty, although they need to be 


\begin{tabular}{lcccc}
\hline & \multicolumn{2}{c}{ Epi } & \multicolumn{2}{c}{ Endo } \\
& CC & RE & CC & RE \\
\hline MFS & 0.52 & 0.31 & - & - \\
VM & 0.58 & 0.28 & 0.60 & 0.30 \\
\hline
\end{tabular}

Table 2. CC and RE of the MFS and VM, four pacing sites, left ventricular scar.

A
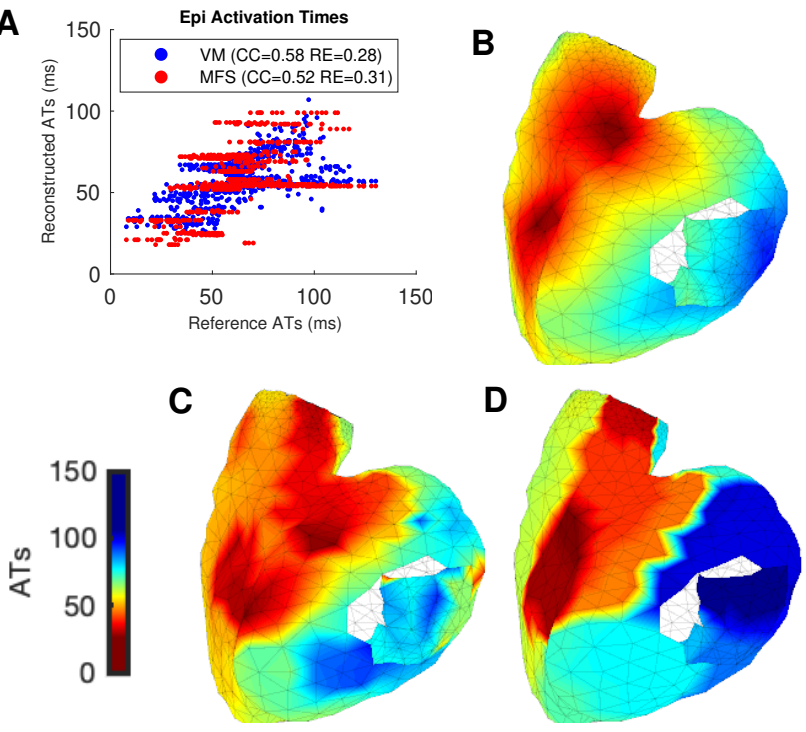

Figure 4. Epicardium, four pacing sites, left ventricular scar. The scar region is cut out. A: reconstructed ATs with respect to reference ATs, MFS in red and VM in blue. B: simulated ATs. C: ATs reconstructed by VM. D: ATs reconstructed by MFS.

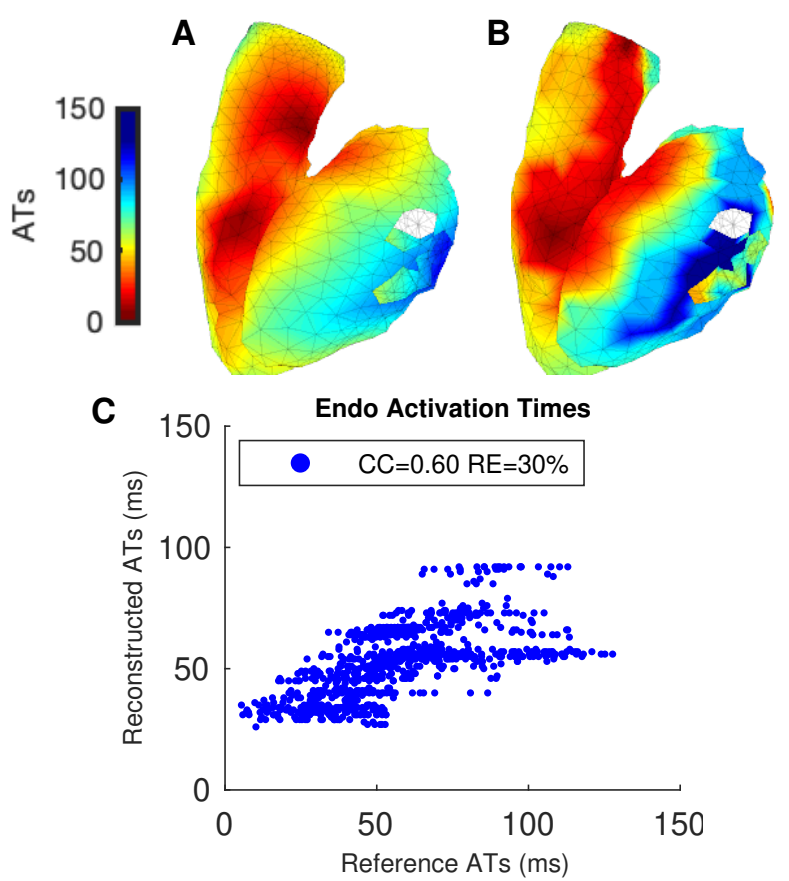

Figure 5. Endocardium, four pacing sites, left ventricular scar. The scar region is cut out. A: simulated ATs. B: VM reconstructed ATs. C: reconstructed ATs with respect to reference ATs.

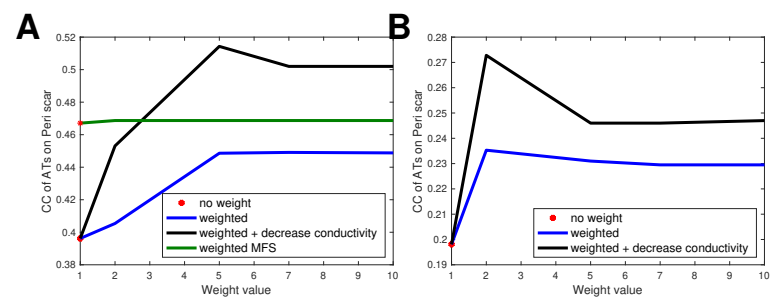

Figure 6. CC only on the neighborhood of the scar, four pacing sites, left ventricular scar. CC for $1 \leq w \leq 10$ (MFS in green, VM with $\rho=1$ in blue, VM with $\rho=0.1$ in black). A: Epicardium. B: Endocardium

confirmed, e.g. by studying more computational examples, and by applying it to experimental or clinical data. Many possible improvements remain, in the spatial distribution of the weights, or the value of the conductivity in the scar.

\section{Acknowledgments}

This study received financial support from the French Government as part of the "Investments for the $\mathrm{Fu}$ ture" program managed by the National Research Agency (ANR), Grant reference ANR-10-IAHU-04, and also from European Research Council (ERC), Grant ECSTATIC (715093). This work was granted access to the HPC resources of CINES under the allocation 2020A0070307379 made by GENCI.

\section{References}

[1] Wang Y, Rudy Y. Application of the method of fundamental solutions to potential-based inverse electrocardiography. Annals of Biomedical Engineering 2006;34(8):1272-1288.

[2] Potse M. Scalable and accurate ECG simulation for reactiondiffusion models of the human heart. Frontiers in Physiology 2018;9:370.

[3] Hecht F. New development in freefem++. Journal of Numerical Mathematics 2012;20(3-4):251 - 266.

[4] Duchateau J, Potse M, Dubois R. Spatially coherent activation maps for electrocardiographic imaging. IEEE Transactions on Biomedical Engineering 2017;64(5):1149-1156.

Address for correspondence:

Mohamadou Malal Diallo

Inria Bordeaux Sud-Ouest, 200 avenue de la vieille tour, 33405

Talence, France.

mohamadou-malal.diallo@inria.fr. 